\title{
Influence of the inner tilt angle on downwash airflow field of multi-rotor UAV based on wireless wind speed acquisition system
}

\author{
Ling Wang*, Qihang Hou, Junpeng Wang, Zhiwei Wang, Shumao Wang \\ (Beijing Key Laboratory of Optimized Design for Modern Agricultural Equipment, College of Engineering, \\ China Agricultural University, Beijing 100083, China)
}

\begin{abstract}
The downwash airflow field is an important factor influencing the spraying performance of plant protection UAV, and the structural design of rotors directly affects the characteristics of the downwash airflow field. Therefore, in this study, three-dimensional models of a six-rotor UAV with various inner tilt angles were established to simulate and analyze the influence of the inner tilt angle on the downwash airflow field based on the Reynolds average NS equation, RNG $k-\varepsilon$ turbulence model, etc.. On this basis, a wireless wind speed acquisition system using the TCP server was developed to carry out the test through the marked points with real-time detection. The simulation results show that, the variation of inner tilt angles of the six-rotor UAV did not cause significant difference in the time dimension of the downwash airflow field, and with the change of the inner tilt angle from $0^{\circ}$ to $8^{\circ}$, the distribution of downwash airflow field tended to obliquely shrink towards the central axis direction, and the amplitude of linear attenuation of airflow speed was also increased, which the difference of attenuation amplitude was $1 \mathrm{~m} / \mathrm{s}$. Besides, under the different inner tilt angles, the airflow velocity in "lead in area" was significantly greater than that in the "lead out area", and the difference of air velocity distribution in space would affect the uniformity of droplet deposition. Through the calibration test, the measurement accuracy error of the developed system was lower than $0.3 \mathrm{~m} / \mathrm{s}$, and the adjusted $R^{2}$ of the calibration fitting equation was higher than 0.99 . The test and simulation values at test points from $0.2-2.3 \mathrm{~m}$ below the rotors exhibit the same variation trend, and the average relative error at the height of 1.1-2.3 $\mathrm{m}$ below the rotors and 0.2-0.8 m near the ground was within $10 \%$ and $20 \%$, respectively. The simulation and test results were highly reliable, which could provide basis and reference for the design and optimization of plant protection drones.
\end{abstract}

Keywords: plant protection UAV, downwash airflow field, inner tilt angle, numerical simulation, wind speed acquisition DOI: $10.25165 /$ j.ijabe.20211406.6477

Citation: Wang L, Hou Q H, Wang J P, Wang Z W, Wang S M. Influence of inner tilt angle on downwash airflow field of multi-rotor UAV based on wireless wind speed acquisition system. Int J Agric \& Biol Eng, 2021; 14(6): 19-26.

\section{Introduction}

Plant protection using unmanned aerial vehicle (UAV) has gradually become an important development trend of aerial pesticide application due to low operating costs, flexible operations, strong automatic control capabilities, and no need for dedicated take-off and landing sites ${ }^{[1]}$, and it is widely used in East Asian countries such as Japan, China and South Korea for low-altitude and low-volume applications ${ }^{[2]}$. In recent years, relevant studies show that the downwash airflow field of UAV plays an important role in the spraying performance, and the distribution shape and law in the crop canopy ${ }^{[3]}$. Besides, the intensity of the downwash airflow field directly affects the deposition area, penetration ability and deposition rate of the droplets ${ }^{[4]}$.

At present, the research on the downwash airflow field of plant protection UAV mostly focused on flight parameters such as

Received date: 2021-01-22 Accepted date: 2021-06-22

Biographies: Qihang Hou, Postgraduate, research interests: agricultural aviation application technology, Email: wysummer@cau.edu.cn; Junpeng Wang, undergraduate, research interests: Fluid simulation method; Email: 2018307160219@cau.edu.cn; Zhiwei Wang, undergraduate, research interests: Advanced DOE, Email:1149629524@qq.com; Shumao Wang, Professor, research interests: agricultural intelligent equipment, Email: wangshumao@ cau.edu.cn.

*Corresponding author: Ling Wang, $\mathrm{PhD}$, Lecturer, research interests: agricultural aviation application and agricultural intelligent equipment. Room 457, College of Engineering, China Agricultural University, Beijing 100083, China. Tel: +86-13811236360, Email: wangling.0928@163.com. aircraft altitude, flight speed, etc., environmental parameters such as wind speed and direction, environmental temperature and humidity, etc., and spraying parameters such as nozzle position, spray pressure, etc ${ }^{[5-8]}$. In addition, the downwash airflow field of multi-rotor UAV is closely related to the structure and design of the UAV itself. The YMR-08 eight-rotor plant protection UAV launched by Yamaha in 2018 used dual nozzles and a conventional six-rotor body structure, and the two nozzles were designed as a reversed double propeller, which greatly improved the flow speed of the downwash airflow ${ }^{[9]}$. The T30 plant protection UAV launched by DJI in 2020 adopted a deformed wing structure, and during the field operation, the arms of No.1 and No.4 were raised and tilted inward to increase pesticides deposition in the middle and lower part of the tree crown and the back of the leaves ${ }^{[10]}$. The V40 plant protection UAV launched by JIFEI Technology in 2020 adopted a tilting dual-rotor structure. Since only the angle of the tilting dual-rotor needed to be adjusted, the flight of V40 (including acceleration, emergency stop, etc.) was more flexible. The tilting double-rotor structure also brought a strong, concentrated and stable wind field, and the penetration power of pesticide application was increased to more than twice that of traditional plant protection using $\mathrm{UAV}^{[11]}$. Therefore, the structure and design parameters of $\mathrm{UAV}$, especially the inner tilt angle has an influence on the distribution law of the downwash airflow field, and the droplet deposition effect will also be affected due to the changing of the downwash airflow field ${ }^{[12,13]}$.

Besides, accurate and real-time field tests are necessary to study the distribution law of downwash airflow field of UAV. 
Wang et al. ${ }^{[14]}$ used a wireless impeller wind speed sensor network measurement system to study the wind field of an unmanned oil-powered single-rotor helicopter flying in a rice field. The mechanical impeller anemometer mainly relied on the downwash airflow of UAV to rotate the impeller and thus generated electromagnetic signals, which in return gave feedback on wind speed. Wu et al. ${ }^{[15]}$ proposed a flexible polypropylene detection device based on the principle of full bridge strain effect to realize on-line rapid detection of the downwash flow field of a multi-rotor UAV. Tang et al. ${ }^{[16]}$ developed an ultrasonic anemometer array and data acquisition software for high-precision measurement of the downwash airflow of UAV. Li et al. ${ }^{[17]}$ used the S Pitot tube sensor array to obtain the vertical wind speed and explored the interaction between the rice canopy and the rotor wind field. Kompenhans et al. ${ }^{[18]}$ first proposed the Particle Image Velocimetry (PIV) to measure the rotor wind field. In general, most of the existing wind field testing equipment for agricultural UAV were built based on the principle of the mechanical impeller which the wind speed sensors were fixed on a tripod to perform multi-point detection, and it had a large shape and interference to the wind field measurement which affected the accuracy of the wind speed measurement. Compared to the mechanical measurement method, ultrasonic and pitot tube wind speed measurement methods had few applications in downwash airflow measurement.

In this study, a six-rotor plant protection UAV was used as the test object to study the distribution characteristics of the downwash airflow field with various inner tilt angles. The three-dimensional models with inner tilt angles of $0^{\circ}, 2^{\circ}, 4^{\circ}, 6^{\circ}$, and $8^{\circ}$ were established using fluid simulation on Reynolds average NS equation, RNG turbulence model, etc. On this basis, a wireless wind speed acquisition system based on thermosensitive sensor was developed using the TCP server to accurately and wirelessly measure the wind speed in real-time, and the wind speed test of the downwash airflow field was carried out through the marked points. The simulation and test research were to provide the basis and reference for the design and optimization of plant protection UAV.

\section{Materials and methods}

\subsection{Numerical simulation method}

In this study, the DJI M600 six-rotor UAV is selected as the research model. As shown in Figure 1a, the UAV arm is tilted inward as a whole, and the angle between the arm and the horizontal plane is the inner tilt angle, which is set to $0^{\circ}, 2^{\circ}, 4^{\circ}, 6^{\circ}$, $8^{\circ}$, respectively. The other parameters of the six-rotor UAV model are kept unchanged. Besides, the UAV has three counter-clockwise positive rotors (rotor 1 , rotor 3 , and rotor 5) and three clockwise anti-rotors (rotor 2 , rotor 4 , and rotor 6); the layout of the rotors is shown in Figure 1b. The wheelbase is $1133 \mathrm{~mm}$, the two rotors are distributed alternately, and the angle between adjacent rotors is $60^{\circ}$.

In order to accurately simulate the distribution law of the downwash airflow field of six-rotor UAV, it is particularly important to accurately construct the three-dimensional model of the forward and reverse rotors. The contactless 3D scanner PTOP300B (Tianjin Weisen General Technology Co., Ltd.) is used to scan the forward and reverse rotors of DJM600, and the point cloud data generated by scanning software Techlego is imported into GEOMAGIC STUDIO for data processing. Finally, the GEOMAGIC Design software is used to realize the reverse construction of the model. The final rotor model is shown in Figure 2, with a rotor diameter of $533 \mathrm{~mm}$.

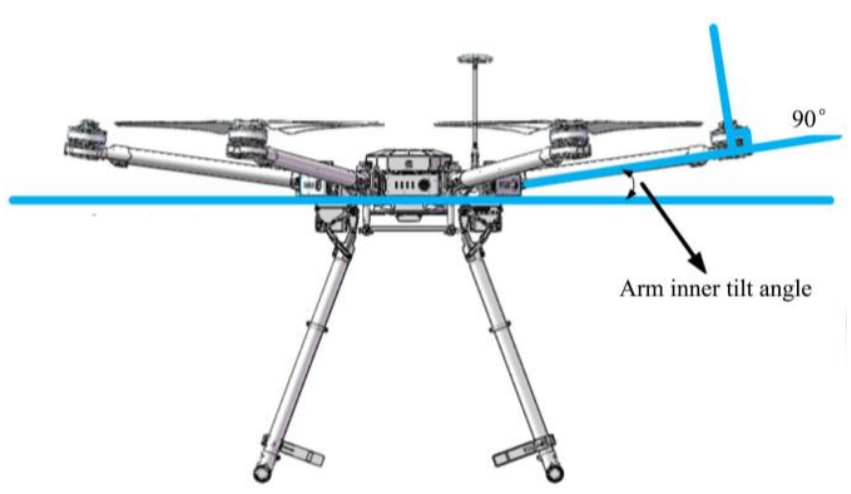

a. Arm inner tilt angel

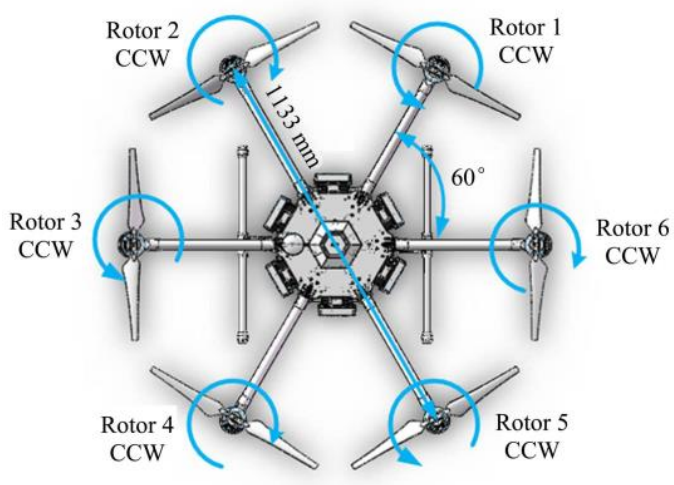

b. Rotors layout

Figure 1 Definition of arm inner tilt angel and rotors layout of the DJI M600 six-rotor UAV

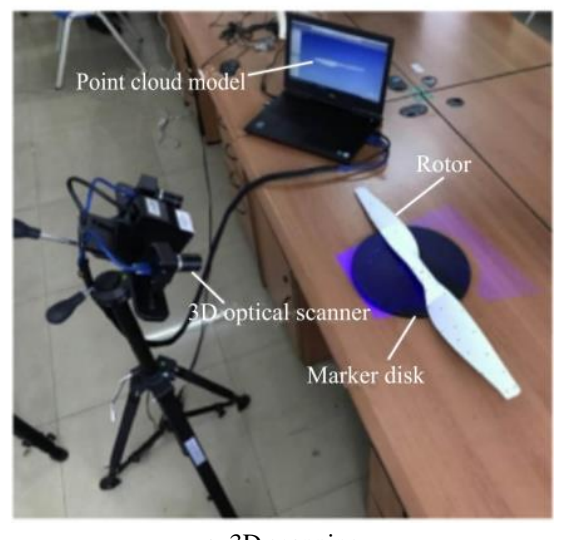

a. 3D scanning

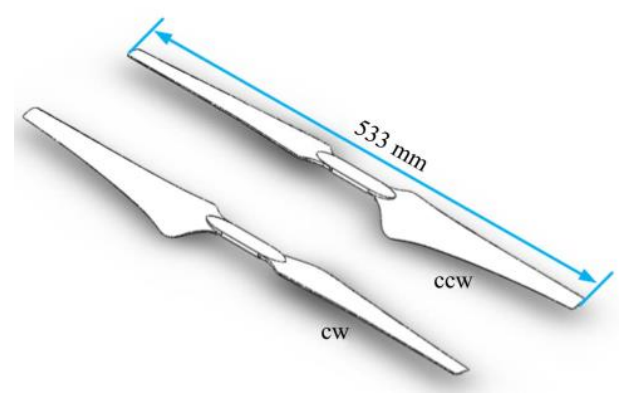

b. Geometric models

Figure 2 3D scanning and geometric models of rotors

The UAV is reasonably simplified, and only the rotor part is retained when processing the six-rotor UAV model, which makes the numerical simulation solution more accurate ${ }^{[19]}$. In this paper, the total circumference of the rotation domain is the air stationary domain, with a length, width and height of $5 \mathrm{~m} \times 5 \mathrm{~m} \times 3.94 \mathrm{~m}$ (Figure 3). The six rotors constitute six rotation domains, each of which is $2.44 \mathrm{~m}$ above the ground, with a diameter of $552 \mathrm{~mm}$, a height of $28 \mathrm{~mm}$ and a rotating speed of $1700 \mathrm{r} / \mathrm{min}$. There are 
seven computational domains in the simulation experiment, and the rotating domain and the static domain exchange information through the interface surface. The lower boundary of the static domain is the ground, the upper boundary is the velocity inlet, and the side is the pressure outlet.

The downwash airflow field of six-rotor UAV is turbulent shear flow, and the RNG turbulence model is selected for simulation. The finite volume method is used to discretize the calculation domain. Due to the low airflow rate, it is assumed that the air is not compressible. The pressure solver and SIMPLE algorithm are used to iteratively calculate the discrete control equations $^{[20-22]}$, with a time step length of $0.025 \mathrm{~s}$ and 30 iterations for each step length, and the total number of steps are 2000 .

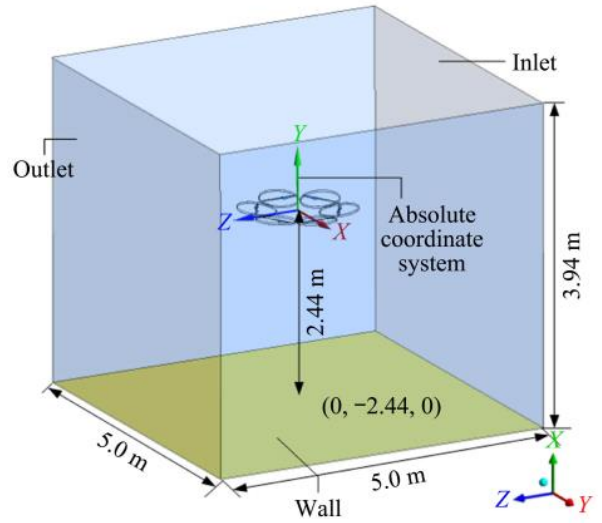

Figure 3 Schematic diagram of calculation region

\subsection{Wireless wind speed acquisition system and test design}

To detect the wind speed of downwash airflow field, a wireless wind speed acquisition system is developed shown in Figure 4a. The wind speed acquisition system adopts micro wireless wind speed acquisition network, which is composed of wind speed acquisition node, a relay router and a TCP server. The wind speed acquisition node uses a thermosensitive wind speed probe, which has a small size of $7.20 \mathrm{~mm} \times 2.40 \mathrm{~mm} \times 0.15 \mathrm{~mm}$. The thermal wind speed probe is fixed in a cylindrical shell with a rectangular air guide hole, and the probe is located in the center of the guide hole. The wind speed acquisition node which is $30 \mathrm{~mm} \times 20$ $\mathrm{mm} \times 35 \mathrm{~mm}$ in size is consisted of the probe, the signal acquisition module, conditioning module, power supply module and wireless communication module. The volume is small and it can reduce the interference of the wind speed collection module on the wind field. Besides, the range of measurement of wind speed is 0.1$30 \mathrm{~m} / \mathrm{s}$, the measurement accuracy is 0.03 , the sampling frequency is $20 \mathrm{~Hz}$ and the response speed is $0.1 \mathrm{~s}$.

The micro wireless wind speed acquisition network adopts a star-shaped network topology structure. A local area network is established through a relay router, to which the client and server are directly connected, and the client and server exchange data through the relay router. Based on the TCP server, the wind speed acquisition node sends the data packet wirelessly to the server, and through the PC software, the server completes the data parsing, display and storage of the wind speed, etc. The design concept of the software is shown in Figure 4b. The wind speed acquisition network can simultaneously realize the cyclic acquisition, wireless transmission and real-time processing of 20 channels.

In order to verify the accuracy of the numerical simulation, the six-rotor plant protection UAV is fixed on the bracket, and the wind speed test is carried out at each space mark point under the rotor. The wind speed measurement points are arranged directly below the center of each rotor. The distance between two adjacent measurement points in the vertical direction is $0.3 \mathrm{~m}$. From $0.2 \mathrm{~m}$ to $2.3 \mathrm{~m}$ above the ground, a total of 8 measurement heights are divided, each of which has its own 6 measuring points. There are a total of 48 measuring points in the test (as shown in Figure 5), and each measuring point is tested in the vertical direction in turn.

During the test, when the wind speed of UAV stabilizes, it starts timing. The wind speed collection node transmits data every $0.05 \mathrm{~s}$, each measurement point is tested for $6 \mathrm{~s}$, and 120 wind speed data are recorded. There is no natural wind during the test, the indoor temperature is $29.5^{\circ} \mathrm{C}$, and the humidity is $65 \%$. After the test, the data of each measuring point are processed on average.

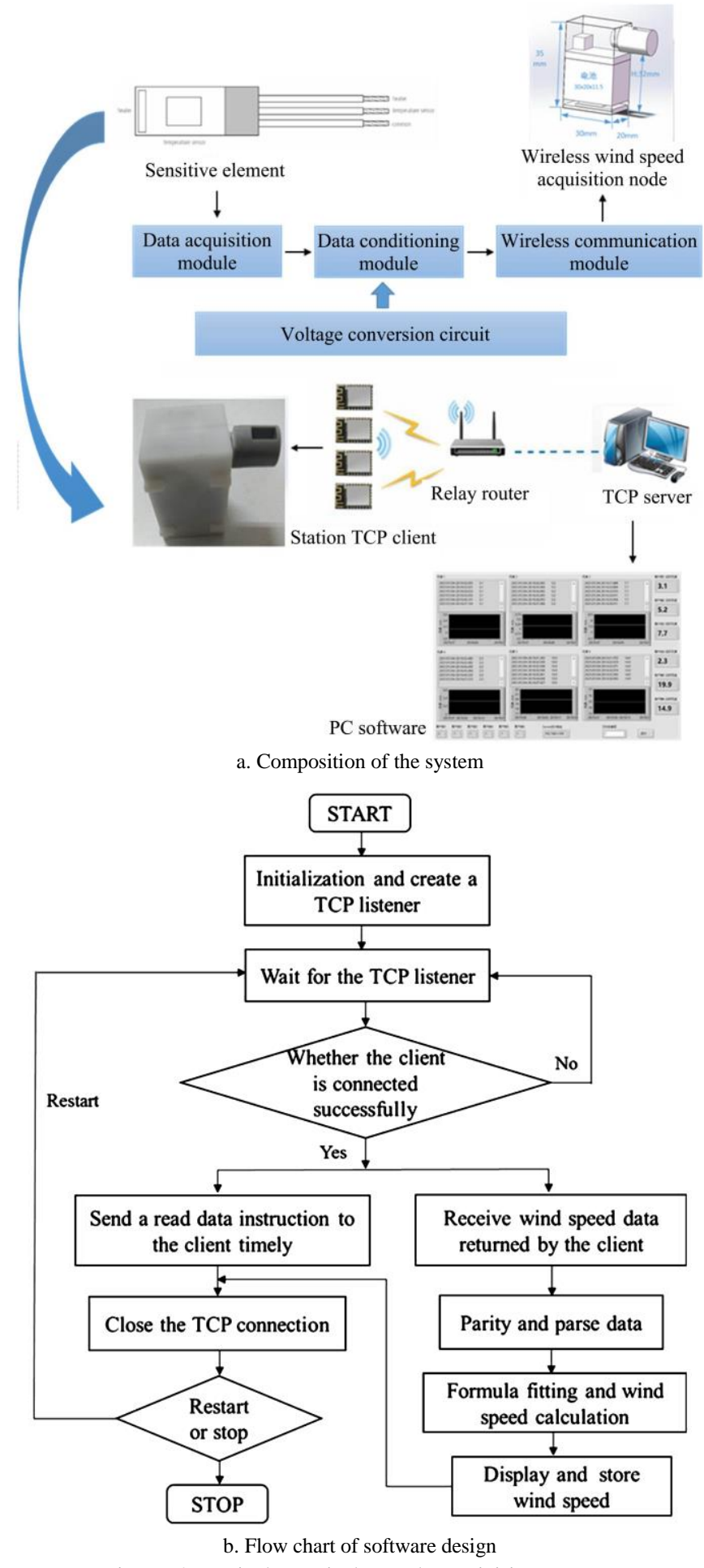

Figure 4 Wireless wind speed acquisition system 

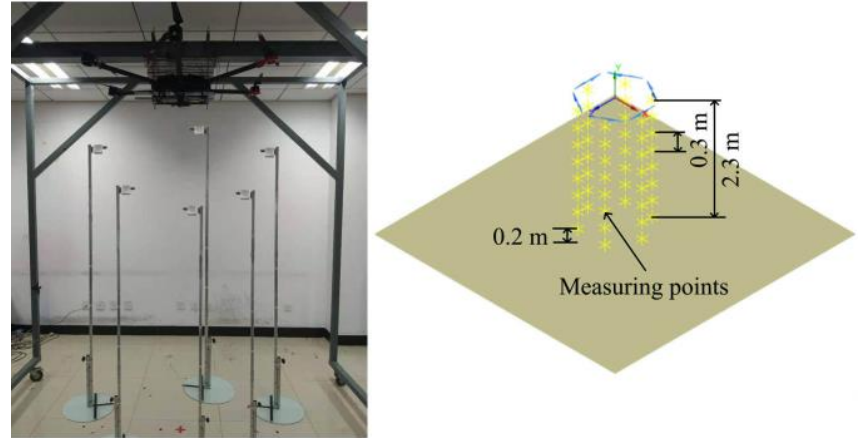

Figure 5 Schematic diagram of measuring points and UAV test bench

\section{Analysis of numerical simulation results}

\subsection{Law of downwash airflow field in time dimension}

For analyzing the downwash airflow field of various inner tilt angles in time dimension, simulation data within $5 \mathrm{~s}$ are collected. Figure 6 shows that the law of downwash airflow field was basically the same with various inner tile angles at the same rotational speed and height. From the moment when the rotor starts to rotate, the downwash airflow generated by the rotor develops to a height of about $1.5 \mathrm{~m}$ above the ground after $0.5 \mathrm{~s}$, and touch the ground at the moment of $1.5 \mathrm{~s}$. At $2 \mathrm{~s}$, the downwash airflow hits the ground and tends to be stable in the vertical direction, dispersing in the surrounding areas on the ground. At the moment of $3 \mathrm{~s}$, the downwash airflow basically reaches a stable state in the vertical and horizontal directions. At the same time, due to air resistance and ground effect, it can be found that the airflow forms a vortex on the ground, which rises slightly. In the subsequent period of time, the airflow will still be hoisted on the ground.
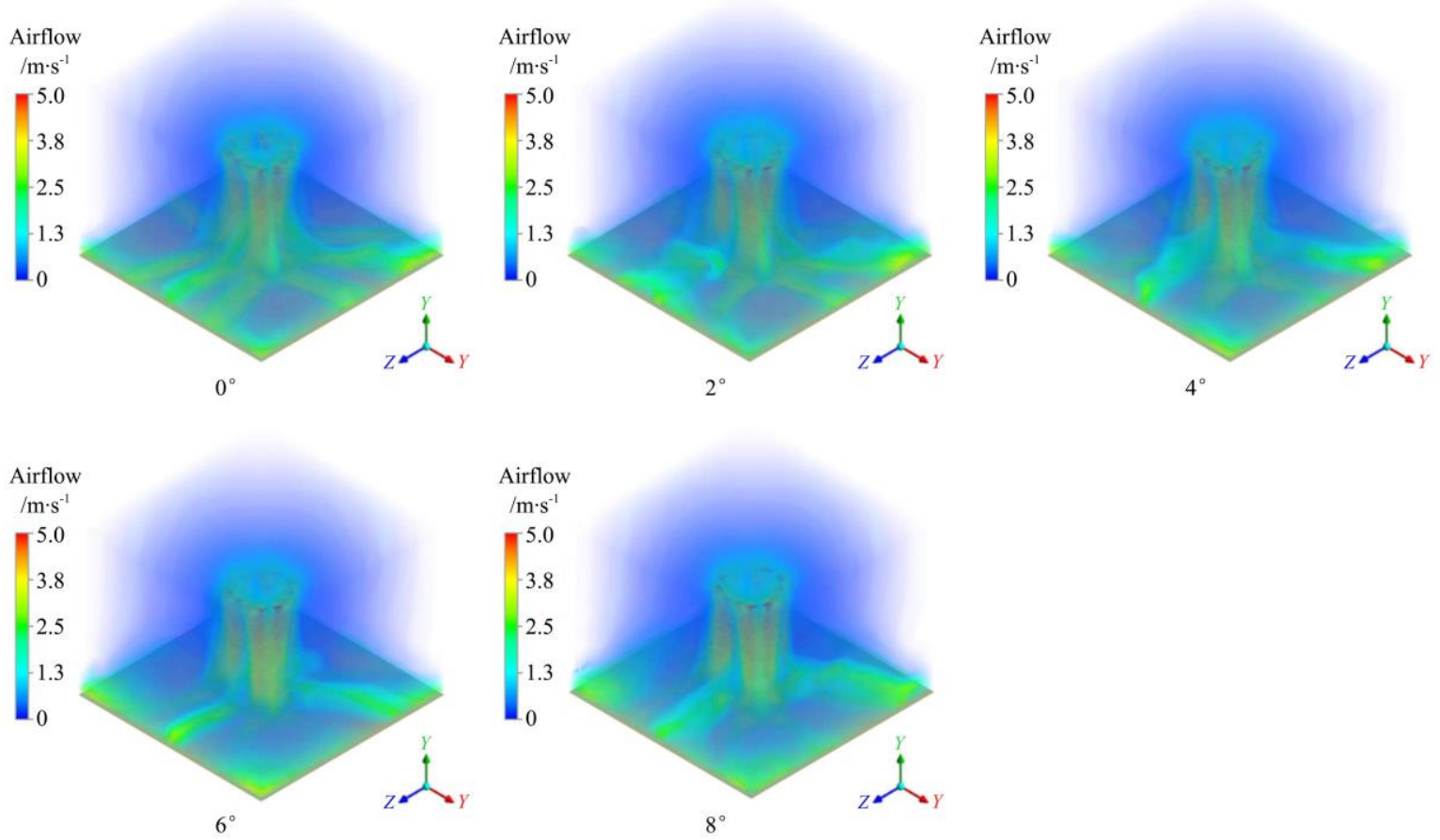

Figure 6 Distribution of downwash airflow field of six rotor UAV with various inner tilt angles at $3 \mathrm{~s}$

\subsection{Law of downwash flow field in spatial dimension}

Figure 7 shows the law of downwash airflow field in spatial dimension, and it is the velocity cloud diagram of the $Y O X$ section in the simulation of various inner tilt angles within $5 \mathrm{~s}$. It can be found that it flows downward in the form of six columnar airflows, and due to the interaction of the airflow between the rotors and the influence of atmospheric pressure, the high-speed airflow will obliquely contract toward the central axis of the six rotors (the $Y$ axis of the absolute coordinate system), and the six cylindrical airflows gradually develop to the ground in an inverted cone. With the gradual increase of the inner tilt angle from $0^{\circ}$ to $8^{\circ}$, the degree of oblique contraction of the downwash airflow will gradually decrease. When the angle is $8^{\circ}$, the downwash airflow will no longer obliquely contract and develop vertically to the ground (marked with red dashed line in Figure 7). In addition, the oblique contraction degree of the downwash airflow is weakened, and the middle area of the six columnar airflows will also increase with the variation of the inner tilt angle (marked by the black dotted line in Figure 7). The airflow velocity in this area is low, and the direction of the airflow is irregular. The airflow in the upper part of the area flows downward, and the airflow in the lower part of the area flows upward due to the effect of the ground and air pressure. 3.2.1 Analysis of changes in airflow velocity under each rotor

The change curve of the airflow velocity under each rotor along the $Y$-axis at the time of $3 \mathrm{~s}$ is shown in Figure 8 . There is one obvious difference between the velocity graph with an inner tilt angle of $0^{\circ}$ and the other four velocity graphs: in the interval of $Y=$ $-0.7 \mathrm{~m}$ to $-2 \mathrm{~m}$, the velocity graph with an inner tilt angle of $0^{\circ}$ is concave. As mentioned above, the six columnar airflows obliquely contracted towards the central axis during the flow to the ground. However, the sampling line is a vertical straight line, so the effective airflow velocity is not collected by the sampling line in the range from $-0.7 \mathrm{~m}$ to $-2.0 \mathrm{~m}$, leading to great changes in the velocity curve with the inner tilt angle of $0^{\circ}$ in this interval. In the interval from $Y=-2.00$ to $-2.44 \mathrm{~m}$, the velocity curve fluctuates to varying degrees, and the change rule of the velocity curve is no longer regular. The reason is that the downwash airflow is very close to the ground at this time, the downwash airflow that previously reached the ground spreads and rolls up on the ground, and the airflow near the ground is in a state of dynamic fluctuation.

From $2^{\circ}$ to $8^{\circ}$ of the inner tilt angle, the downwash airflow velocity changes basically the same: the downwash airflow 
increases linearly from the center of the rotor at a speed of $0 \mathrm{~m} / \mathrm{s}$, and the speed reaches a maximum value of $4.2 \mathrm{~m} / \mathrm{s}$ at $0.3 \mathrm{~m}$ below the rotor. Then the airflow velocity begins to decrease linearly, and quickly decays to $0 \mathrm{~m} / \mathrm{s}$ when it is about to touch the ground. As the inner tilt angle varies, the linear decrease of the airflow will gradually increase, and the position of the speed change node will also move back. For example, when the angle is $2^{\circ}$, the airflow drops to $3.8 \mathrm{~m} / \mathrm{s}$ at $Y=-1.70 \mathrm{~m}$, and then attenuates to $0 \mathrm{~m} / \mathrm{s}$ from -1.70 to $-2.44 \mathrm{~m}$. when the angle is $8^{\circ}$, the airflow drops to 2.60 $\mathrm{m} / \mathrm{s}$ at $Y=-2.30 \mathrm{~m}$, and then rapidly decays to $0 \mathrm{~m} / \mathrm{s}$.

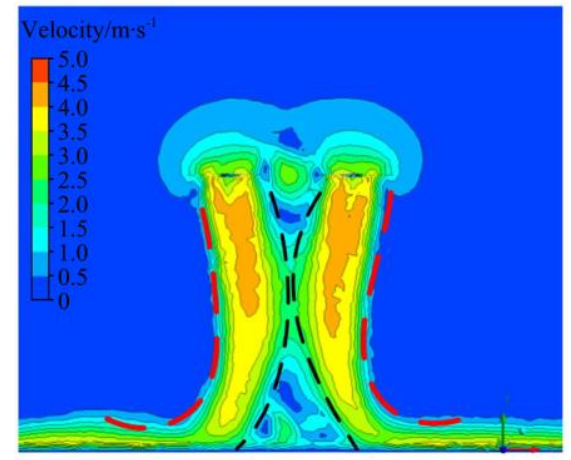

$0^{\circ}$

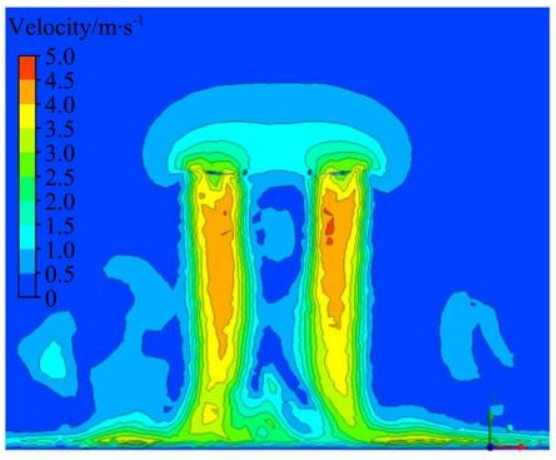

$6^{\circ}$

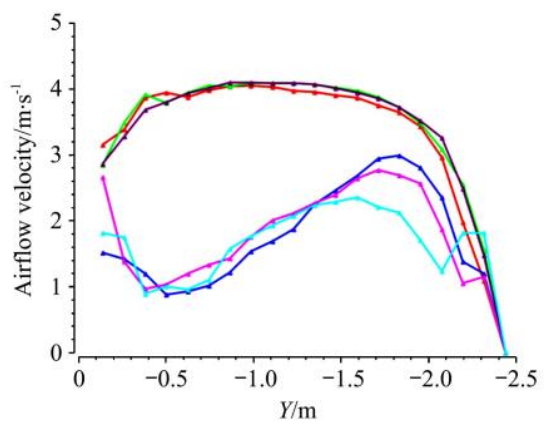

$0^{\circ}$

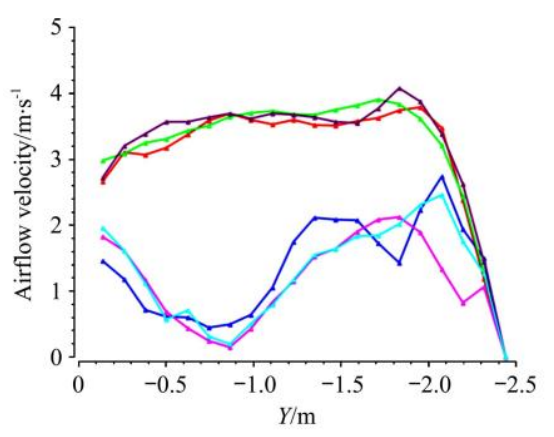

$6^{\circ}$

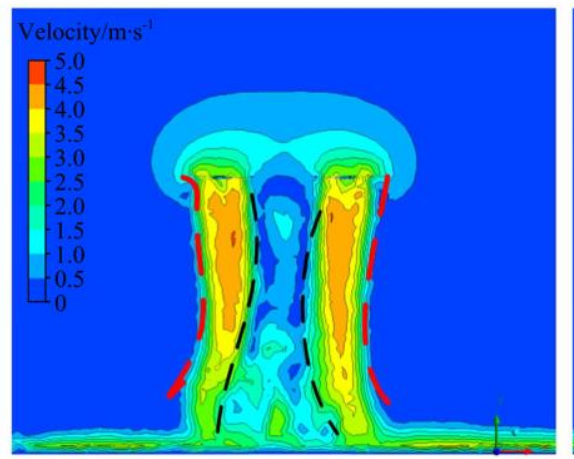

$2^{\circ}$

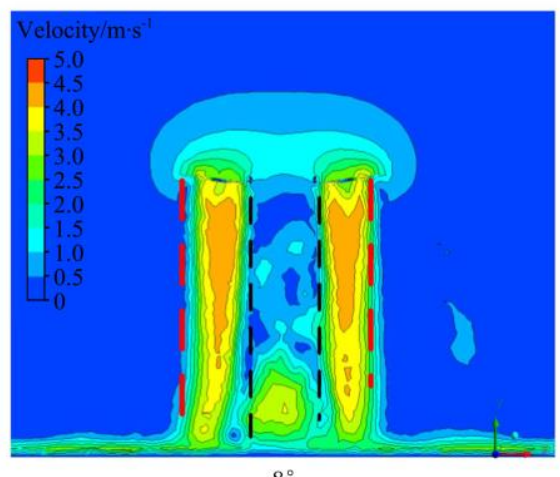

$8^{\circ}$

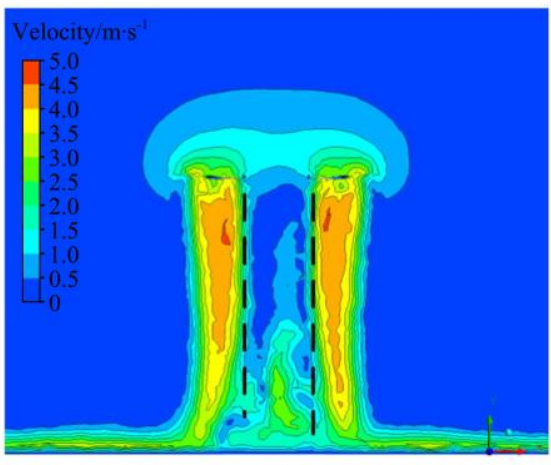

$4^{\circ}$

Figure 7 Velocity reprogram of $Y O X$ section at $5 \mathrm{~s}$

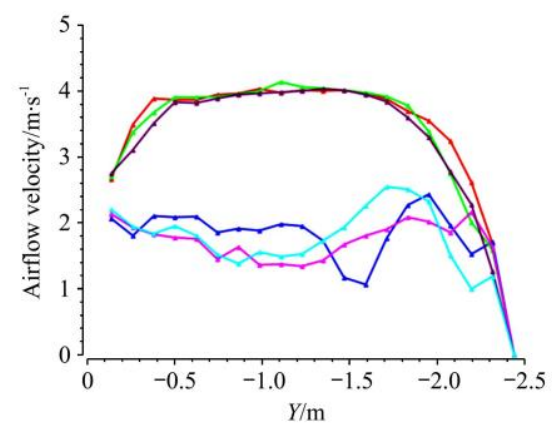

$2^{\circ}$

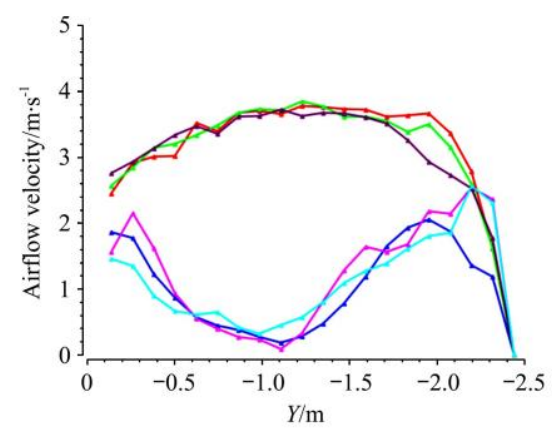

$8^{\circ}$

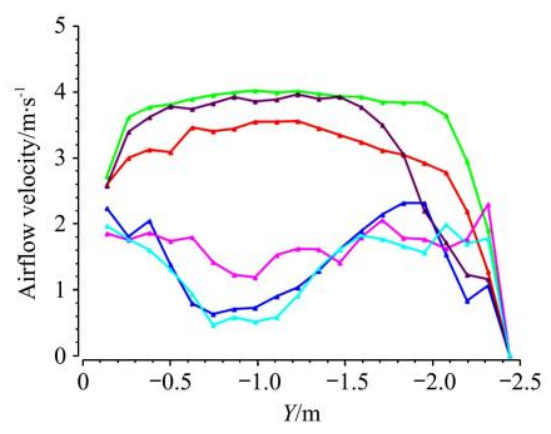

$4^{\circ}$

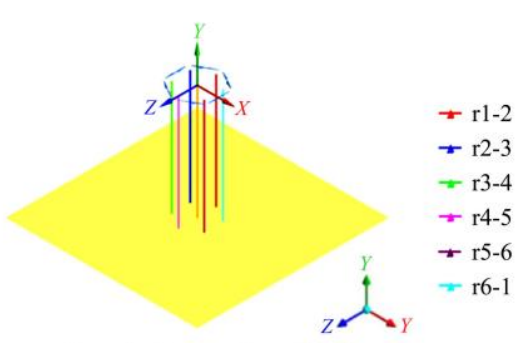

Schematic diagram of sampling line

Figure 8 Change curve of airflow velocity under the center of each rotor at $3 \mathrm{~s}$

\subsubsection{Analysis of changes in airflow velocity between each rotor}

Depending on the direction of rotation of the adjacent rotors, the airflow between the rotors has an "lead in area" or "lead out area", which will affect the distribution and drift of the droplet particles in the horizontal direction ${ }^{[23,24]}$. It has three "lead in area" and three "lead out area" of the six-rotor UAV. In order to accurately and comprehensively explore whether there is a difference between downwash airflow fields of UAV with various inner tilt angles and how big the difference is, six sampling lines are drawn in the vertical direction between each pair of adjacent rotors in this section, so as to conduct a quantitative analysis on the size and variation trend of airflow velocity between rotors. In 
Figure 9, it shows the change curve of airflow velocity at the positions of "lead in area" and "lead out area" at the time of $3 \mathrm{~s}$. Six sampling lines are drawn in the vertical direction between the rotors, starting from the sampling line between rotor 1 and rotor 2 , counterclockwise, and they were named r1-2, r2-3, r3-4, r4-5, r5-6 and r6-1 respectively. Among them, r1-2, r3-4 and r5-6 are the

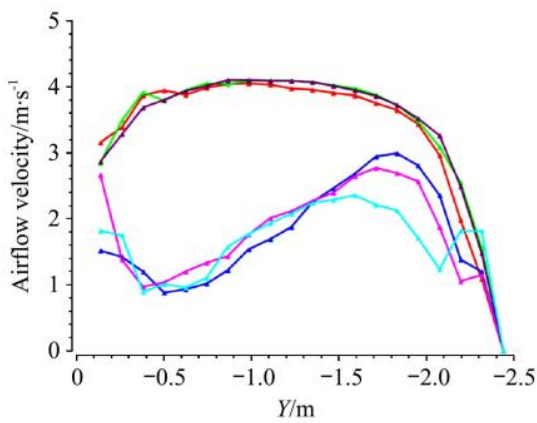

$0^{\circ}$

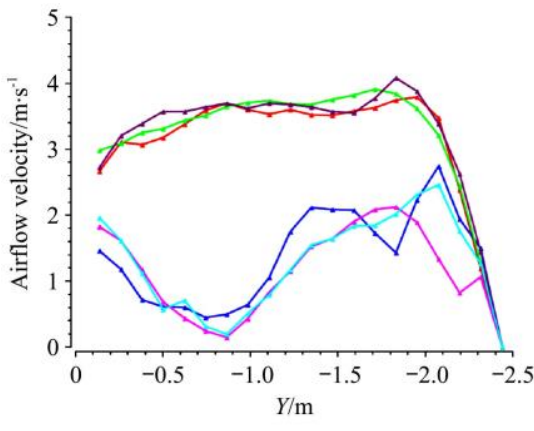

$6^{\circ}$

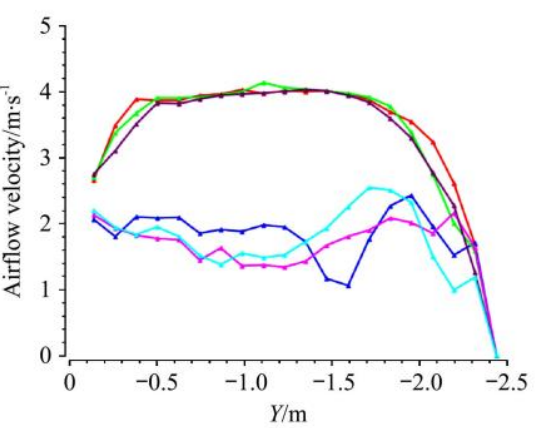

$2^{\circ}$

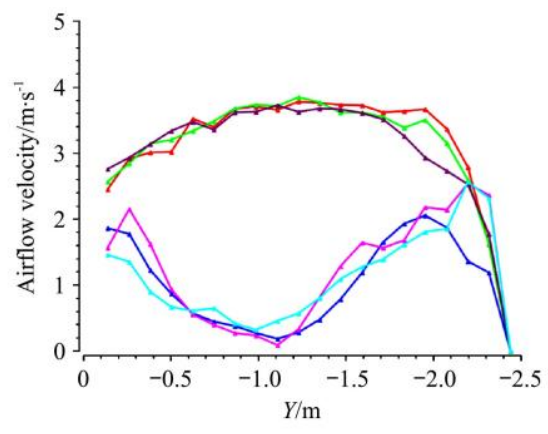

$8^{\circ}$ sampling lines of the three "lead in area", and r2-3, r4-5 and r6-1 are the sampling lines of the three "lead out area" respectively. It can be found that the airflow velocity in the "lead in area" is obviously higher than that in the "lead out area", and it is basically the same until the airflow is near the ground, and suddenly decays to $0 \mathrm{~m} / \mathrm{s}$.

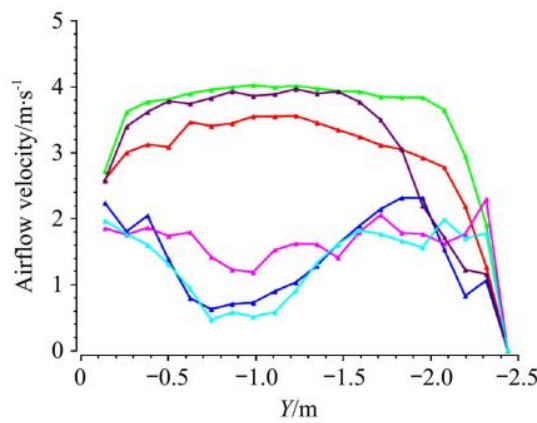

4

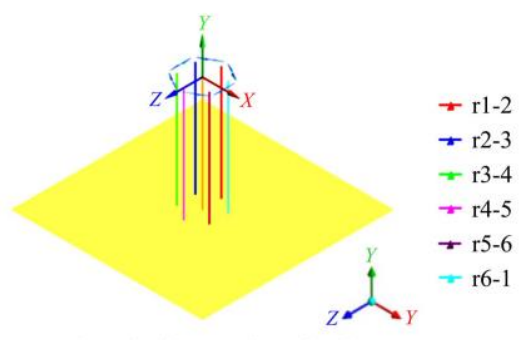

Schematic diagram of sampling line

Figure 9 Change curve of airflow velocity in the "lead in area" and "lead out area" at $3 \mathrm{~s}$

The phenomenon can be explained through Figure 10 and Figure 11. Figure 10 shows the velocity cloud diagram at $1 \mathrm{~m}$ below the rotor at the moment of $3 \mathrm{~s}$ of the $X O Z$ section and the black dashed line corresponds to the position of the $Y O Z$ section in Figure 11. Figure 11 shows the velocity cloud diagram of the $Y O Z$ section in the simulation with an inner tilt angle of $0^{\circ}$ and $8^{\circ}$ at $3 \mathrm{~s}$. The red line on the left is in the "lead out area", and the red line on the right is in the "lead in area".

Due to the different rotation directions of the rotors, under the guidance of the rotors on the airflow, the two adjacent columnar airflows will converge to the "lead in area" and form three larger pairs of airflows, so the "lead in area" has high airflow and velocity. On this basis, it can be seen from the velocity cloud map of the $Y O Z$ section that the velocity distribution on the left and right sides is not symmetrical, and the velocity in the right airflow introduction region is greater than that in the left outflow region.

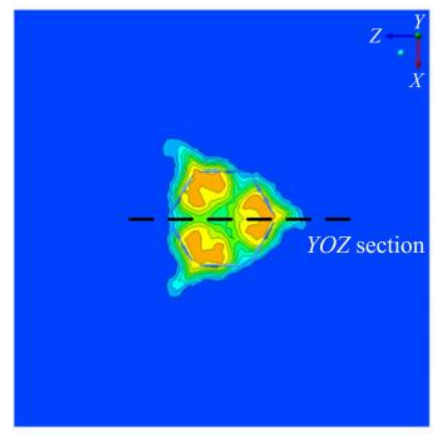

$0^{\circ}$

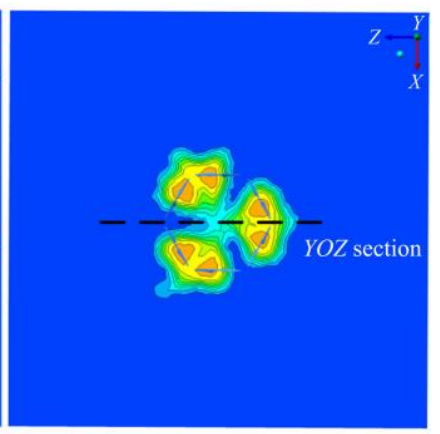

$8^{\circ}$
Figure 10 Velocity nephogram of $X O Z$ section at $1 \mathrm{~m}$ below rotors at $3 \mathrm{~s}$

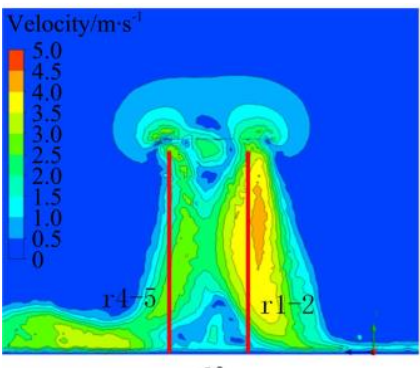

$0^{\circ}$

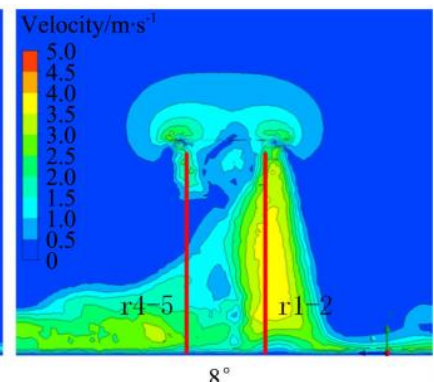

$8^{\circ}$
Figure 11 Velocity nephogram of YOZ section at $3 \mathrm{~s}$

From $0^{\circ}$ to $8^{\circ}$ of inner tilt angle, the change rule of downwash airflow velocity curve in "lead in area" and the position of change node are basically the same. The change rule of the downwash velocity curve in the "lead out area" is also somewhat similar, but the position of the change node is not the same. The difference of airflow velocity in the "lead in area" and "lead out area" will lead to the inhomogeneity of droplet deposition.

\section{Test verification and analysis}

\subsection{Calibration test of the wireless wind speed acquisition system}

The developed wind speed acquisition node is calibrated by the testo-410i anemometer produced by Testo, Germany. The measuring range of the testo- $410 \mathrm{i}$ anemometer is $0.4 \mathrm{~m} / \mathrm{s}$ to $30 \mathrm{~m} / \mathrm{s}$, the measurement accuracy is $\pm 0.2 \mathrm{~m} / \mathrm{s}$, the sampling frequency is $1 \mathrm{~Hz}$, and the resolution is $0.1 \mathrm{~m} / \mathrm{s}$. During the calibration, the wind speed acquisition node is fixed at the center of the low-speed micro wind tunnel experiment section and $20 \mathrm{~cm}$ away from the exit, and the Testo-410i anemometer is used to locate $2 \mathrm{~cm}$ in front of the developed wind speed acquisition node. 


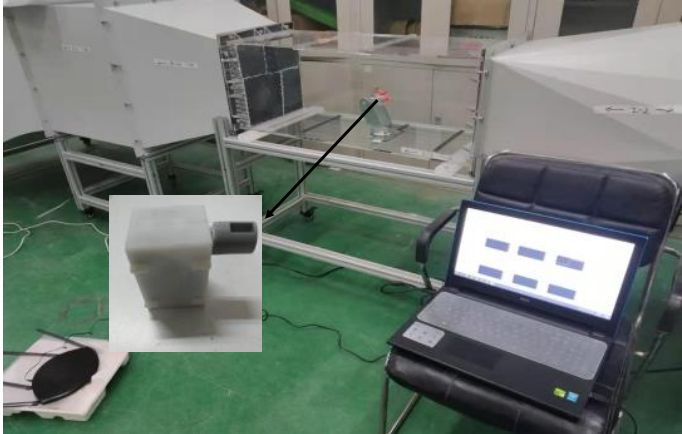

Figure 12 Calibration test device for measured sensor

By adjusting the frequency conversion, the central wind speed of wind tunnel is changed. In the test, the wind speed in the center of the wind tunnel is adjusted to a certain stable value, and 20 groups of data are collected by the Testo-410i anemometer continuously $^{[24]}$. In the meantime, the developed sensor is used to acquire the wind speed in the same situation, and the average value of 20 groups of data is taken as the calibration value. Taking the calibration value of the measured sensor as the abscissa and the average wind speed of the testo-410i anemometer as the ordinate, the data is fitted with a cubic polynomial using the least square method to obtain the calibration curve and calibration equation of the wind speed sensor.

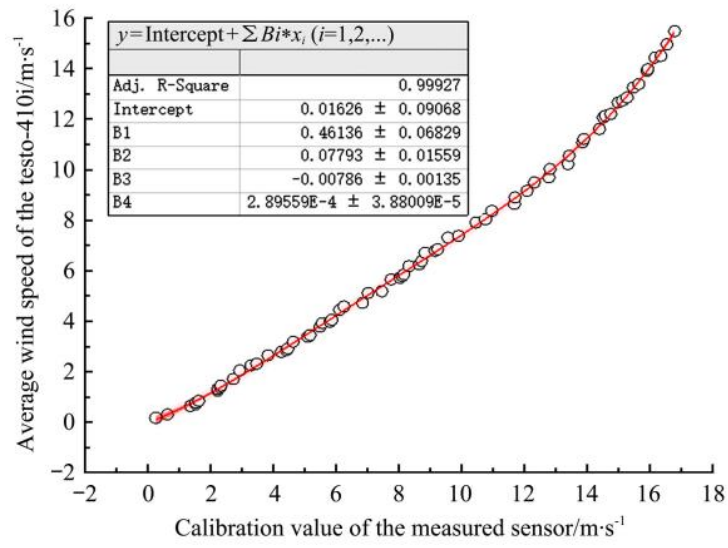

Figure 13 Calibration curve of the measured sensor

The output characteristic of the developed wind speed sensor has a monotonous increasing trend, and the change is relatively small in the range of $0-10 \mathrm{~m} / \mathrm{s}$, with basically linear relationship. In the range of $10-16 \mathrm{~m} / \mathrm{s}$, the change is relatively large with non-linear relationship. Besides, the relative error between the wind speed acquisition node and the wind speed value is \pm 0.03 . By cubic polynomial fitting using least square method, the fitting equation is as follows, and the R Square of the fitting curve is above 0.99 .

$$
y=-0.0078 x^{3}+0.0779 x^{2}+0.4614 x+0.0163
$$

where, $y$ is the wind speed of wind tunnel; $x$ is the wind speed acquired by the developed sensor.

\subsection{Validation and analysis with the inner tilt angle of $8^{\circ}$}

Through the establishment of a UAV wind speed test bench, the wireless wind speed collection network is used to test the wind speed at different heights under the six rotors of the UAV. The simulation data at $3 \mathrm{~s}$ are selected for comparison with the experimental data.

$$
e=\frac{\left|V_{s}-V_{t}\right|}{V_{t}}
$$

where, $V s$ is simulated velocity, $\mathrm{m} / \mathrm{s} ; V t$ is tested velocity, $\mathrm{m} / \mathrm{s} ; e$ is the relative error between simulated and tested values, $\%$.
From Table 1, it can be found that the test value and simulation value of downwash airflow at each measuring point maintain the same trend, and in each measuring point of 1.1-2.3 $\mathrm{m}$ from the ground, the relative error between the simulated data and the test data is less than $8 \%$. In the measurement points of $0.2-0.8 \mathrm{~m}$ away from the ground, the relative error between most of the simulated data and the test data is between $10 \%-30 \%$. At this moment, the downwash airflow is close to the ground, and eddy currents will be generated due to ground effect in both numerical simulation and actual test, leading to the flow field disorder near the ground and difference of simulation and test data. In general, the relative error between the simulated data and the test data is accurate and acceptable.

\begin{tabular}{|c|c|c|c|c|c|c|c|c|c|}
\hline \multirow{2}{*}{ Position } & \multirow{2}{*}{ Value } & \multicolumn{8}{|c|}{ Measuring height $/ \mathrm{m}$} \\
\hline & & 2.3 & 2.0 & 1.7 & 1.4 & 1.1 & 0.8 & 0.5 & 0.2 \\
\hline \multirow{3}{*}{ Rotor 1} & $V_{s}$ & 3.6 & 4.1 & 4.0 & 3.8 & 3.8 & 3.0 & 1.7 & 1.6 \\
\hline & $V_{t}$ & 3.7 & 4.0 & 3.8 & 3.6 & 3.5 & 3.5 & 2.4 & 1.8 \\
\hline & $e$ & 2.7 & 2.5 & 5.3 & 5.6 & 8.6 & 14.3 & 29.1 & 11.1 \\
\hline \multirow{3}{*}{ Rotor 2} & $V_{s}$ & 3.3 & 4.2 & 4.1 & 4.0 & 3.7 & 3.4 & 3.0 & 2.3 \\
\hline & $V_{t}$ & 3.5 & 3.9 & 4.2 & 4.3 & 4.0 & 3.7 & 2.8 & 2.1 \\
\hline & $e$ & 5.7 & 7.7 & 2.4 & 7.0 & 7.5 & 8.1 & 7.1 & 9.5 \\
\hline \multirow{3}{*}{ Rotor 3} & $V_{s}$ & 3.6 & 4.2 & 4.1 & 3.7 & 3.6 & 3.3 & 3.3 & 2.5 \\
\hline & $V_{t}$ & 3.8 & 4.3 & 3.8 & 4.0 & 3.8 & 3.0 & 2.6 & 2.7 \\
\hline & $e$ & 5.3 & 2.3 & 7.9 & 7.5 & 5.3 & 10.0 & 26.9 & 7.4 \\
\hline \multirow{3}{*}{ Rotor 4} & $V_{s}$ & 3.6 & 4.3 & 4.1 & 4.0 & 3.6 & 3.1 & 1.9 & 1.5 \\
\hline & $V_{t}$ & 3.4 & 4.1 & 3.9 & 3.8 & 3.9 & 3.5 & 2.7 & 1.8 \\
\hline & $e$ & 5.9 & 4.9 & 5.1 & 5.3 & 7.7 & 11.4 & 29.6 & 16.6 \\
\hline \multirow{3}{*}{ Rotor 5} & $V_{s}$ & 3.4 & 4.3 & 4.1 & 4.0 & 3.6 & 3.3 & 3.0 & 2.6 \\
\hline & $V_{t}$ & 3.3 & 4.7 & 3.8 & 3.7 & 3.4 & 3.7 & 2.6 & 2.3 \\
\hline & $e$ & 3.0 & 8.5 & 7.9 & 8.1 & 5.9 & 10.8 & 15.4 & 13.0 \\
\hline \multirow{3}{*}{ Rotor 6} & $V_{s}$ & 3.4 & 4.1 & 4.1 & 3.9 & 3.6 & 3.4 & 1.9 & 1.9 \\
\hline & $V_{t}$ & 3.7 & 4.0 & 3.9 & 3.6 & 4.0 & 3.9 & 2.6 & 2.4 \\
\hline & $e$ & 8.1 & 2.5 & 5.1 & 8.3 & 10.0 & 12.8 & 26.9 & 20.8 \\
\hline
\end{tabular}

Table 1 Wind speed value of each measuring point

\section{Conclusions and discussion}

Three-dimensional models of the six-rotor plant protection UAV with inner tilt angle of $0^{\circ}, 2^{\circ}, 4^{\circ}, 6^{\circ}$ and $8^{\circ}$ were established using fluid simulation. The distribution law of the downwash airflow field in time and spatial dimension was numerically simulated, and the distribution characteristics of the airflow field with various inner tilt angles were analyzed. The simulation results showed that: the variation of the inner tilt angle of six-rotor UAV did not cause significant difference in the time dimension of the downwash airflow, and the downwash airflow spread out on the ground at $3 \mathrm{~s}$ and tended to be stable. From $0^{\circ}$ to $8^{\circ}$, as the increase of the inner tilt angle, the downwash airflow tended to obliquely shrink towards the central axis direction, and the amplitude of linear attenuation of airflow velocity was also increased, which the difference of attenuation amplitude was $1 \mathrm{~m} / \mathrm{s}$.

Wireless wind speed acquisition system was designed to realize on-time rapid detection of the downwash airflow field of the six-rotor UAV, and through the marking points, the test of downwash airflow velocity was carried out in the lab. From the calibration and application test, the designed system was reliable and flexible. The measured downwash airflow speed range is $0-30 \mathrm{~m} / \mathrm{s}$ and the relative error is \pm 0.03 .

The test value and simulation value of downwash airflow at 
each measuring point maintained the same trend and the average relative error at the height of 1.1-2.3 m below the rotors and 0.2$0.8 \mathrm{~m}$ near the ground was within $8 \%$ and $20 \%$, respectively. The simulated data and the test data were accurate and acceptable; the influence law of the inner tilt angle on downwash airflow field of six-rotor UAV could provide basis and reference for UAV design and optimization.

In addition, this study is a preliminary step on the influence of inner tilt angle on the downwash airflow field of UAVs, the further exploration subsequently for the droplet deposition effect will be studied.

\section{Acknowledgements}

This work was financially supported by National Natural Science Foundation of China (Grant No. 31801783).

\section{[References]}

[1] Wang L, Lan Y B, Hoffmann W C, Bradley K F, Chen D, Wang S M. Design of variable spraying system and influencing factors on droplets deposition of small UAV. Transactions of the CSAM, 2016; 47(1): 15-22. (in Chinese)

[2] Li J Y, Shi Y Y, Lan Y B, Guo S. Vertical distribution and vortex structure of rotor wind field under the influence of rice canopy. Computers and Electronics in Agriculture, 2019; 159: 140-146.

[3] Chen S D, Lan Y B, Bradley K F, Li J Y, Liu A M, Mao Y D. Effect of wind field below rotor on distribution of aerial spraying droplet deposition by using multi-rotor UAV. Transactions of the CSAM, 2017; 48(8): $105-113$.

[4] Angelis E L D. Stability analysis of a multirotor vehicle hovering condition. Aerospace Science and Technology, 2018; 72: 248-255.

[5] Wang J X, Wang Z, Zhang H, Liu S X, Xing L L, Li Y Y, et al. Optimal experiment of variable spraying parameters for UAV with low volume and low altitude. Jiangsu Agricultural Sciences, 2018; 46(17): 215-219. (in Chinese)

[6] Fritz B K. Meteorological effects on deposition and drift of aerially applied sprays. Trans ASABE, 2006; 49(5): 1295-1301.

[7] Zhu H, Lan Y B, Wu W F, Hoffmann W C, Huang Y B, Xue X Y, et al. Development of a PWM precision spraying controller for unmanned aerial vehicles. Journal of Bionic Engineering, 2010; 7(3): 276-283.

[8] Lan Y B, Thomson S J, Huang Y B, Hoffmann W C, Zhang H H. Current status and future directions of precision aerial application for site-specific crop management in the USA. Computers and Electronics in Agriculture, 2010; 74(1): 34-38.

[9] Lian Q. Study on the variation of downwash and the installation position of nozzles in a six-rotor plant protection drone. Heilongjiang:
Heilongjiang Bayi Agricultural University, 2019. (in Chinese)

[10] Technical parameters of T30 plant protection UAV. http://www.dji.com/cn/t30. 2020.11.

[11] Agricultual UAV of V40. https://www.xa.com/v40-2021. 2021.4.

[12] $\mathrm{Xu} \mathrm{Z}$. SMC based wind disturbance rejection for quad-rotor UAVs. Electronics Optics \& Control, 2017; 24(1): 67-71.

[13] Balaji S, Prabhagaran P, Vijayanandh R, Kumar MS, Kumar RR Comparative computational analysis on high stable hexacopter for long range applications. Lecture Notes in Civil Engineering, 2020; pp. 369-391.

[14] Wang P, Hu L, Zhou Z Y, Yang W S, Liu A M, Luo X W, et a1. Wind field measurement for supplementary pollination in hybrid rice breeding using unmanned gasoline engine single-rotor helicopter. Transactions of the CSAE, 2013; 29(3): 54-61. (in Chinese).

[15] Wu Y L, Qi L J, Zhang H, Musiu E, Yang Z P, Wang P. Design of uav downwash airflow field detection system based on strain effect principle. Sensors, 2019; 19: 2630.

[16] Tang Q, Zhang R R, Ding C, Chen L P, Xu M, Xu G, et al. Application of ultrasonic anemometer array to field measurements of the downwash flow of an agricultural unmanned helicopter. Transactions of the ASABE 2019; 62(5): 1219-1230.

[17] Li J Y., Lan Y B, Wang J W, Chen S D, Huang C, Liu Q, et al. Distribution law of rice pollen in the wind field of small UAV. Int $\mathrm{J}$ Agric \& Biol Eng, 2017; 10(4): 32-40.

[18] Kompenhans J, Raffel M, Vogt A, Fischer M, Bretthauer B, Vollmers H, et al. Investigation of unsteady flow fields in wind tunnels by means of particle image velocimetry. Part of the ERCOFTAC Series book series. 1994

[19] Yang F B, Xue X Y, Zhang L, Sun Z. Numerical simulation and experimental verification on downwash air flow of six-rotor agricultural unmanned aerial vehicle in hover. Int J Agric \& Biol Eng, 2017; 10(4): 21-53.

[20] Yang S H, Tang Q, Zheng Y J, Liu X X, Chen J, Li X L. Model migration for CFD and verification of a six-rotor UAV downwash. Int $\mathrm{J}$ Agric \& Biol Eng, 2020; 13(4): 10-18.

[21] Khalilzadeh A, Ge H, Ng H D. Effect of turbulence modeling schemes on wind-driven rain deposition on a mid-rise building: CFD modeling and validation. Journal of Wind Engineering and Industrial Aerodynamics, 2019; 184: 362-377.

[22] Guo Q W, Zhu Y Z, Tang Y, Hou C J. CFD simulation and experimental verification of the spatial and temporal distributions of the downwash airflow of a quad-rotor agricultural UAV in hover. Computers and Electronics in Agriculture, 2020; 172(4): 105343.

[23] Yang F, Xue X, Cai C, Zhou Q Q. Effect of down wash airflow in hover on droplet motion law for multi-rotor unmanned plant protection machine. Transactions of the CSAE, 2018; 34(2): 64-73. (in Chinese)

[24] Liu H, Chang J L, Hou Z F, Chen Z, Xuan C Z, Shang X B. Investigation on near-surface wireless wind speed profiler based on thermistors. Transactions of the CSAM, 2017; 48(12): 68-77. (in Chinese) 